\title{
Los Procesos de Comunicación Educativos sobre el Cáncer de Próstata.
}

\section{The Educational Communication Processes on Prostate Cancer.}

\author{
Diana del Pilar Baquerizo Villacís ${ }^{1 *(D)}$, Jhonny Amed Vargas Flores ${ }^{1}$, \\ John Chusán Jiménez ${ }^{2}$, Patricio Vega Luzuriaga ${ }^{3}$
}

*Correspondencia:

diana.baquerizo@gmx.es

Teléfono [593] 032498288

Conflicto de intereses: Los autores declaran no tener conflictos de intereses.

Fondos: Ver la página 208

Recibido: 9 Enero 2018

Aceptado: 1 Abril 2018

Publicado: 30 Diciembre 2018

Membrete bibliográfico:

Baquerizo D, Vargas J, Chusán J, Vega P. Los Procesos de Comunicación Educativos sobre el Cáncer de Próstata. Rev. Oncol. Ecu 2018;28(3):202-209.

DOI: https://doi.org/10.33821/240

Copyright Baquerizo, et al. Este artículo es distribuido bajo los términos de Creative Commons Attribution License, el cual permite el uso y redistribución citando la fuente y al autor original.
1. Facultad de Comunicación Social (Facso), Universidad de Guayaquil.

2. Instituto Nacional de Investigación en Salud Pública (INSPI), Centro de Referencia Nacional de Micología, Guayaquil-Ecuador.

3. Instituto Nacional de Investigación en Salud Pública (INSPI), Dirección Técnica de Transferencia del Conocimiento, Guayaquil-Ecuador.

\section{Resumen}

Introducción: El cáncer de próstata es una neoplasia en la cual la comunicación y el conocimiento de la población puede ayudar al diagnóstico temprano y tratamiento temprano. El objetivo de este estudio fue investigar que conocen los pacientes y acompañantes sobre los procesos de comunicación educativa para la salud en cáncer de próstata.

Métodos: El presente estudio descriptivo, fue realizado en el Instituto Oncológico Nacional "Dr Juan Tanca Marengo" Solca-Guayaquil. Se utilizó una encuesta de salud sobre el conocimiento de la entidad nosológica, el conocimiento de los métodos diagnósticos y la predisposición a acudir a charlas educativas. La muestra fue calculada en 80 encuestas a familiares, acompañantes y pacientes del Instituto.

Resultados: Se registraron 80 encuestas. Sobre el cáncer de próstata el $52.5 \%$ de los encuestados declara no tener conocimiento alguno sobre el mismo, un $31.25 \%$ asegura tener poco conocimiento sobre el cáncer de próstata, y el $16.25 \%$ declara tener un conocimiento apropiado sobre el cáncer de próstata. Sobre el diagnóstico de Cáncer de Próstata el $58.75 \%$ de los encuestados no sabe cómo se diagnostica, un $22.5 \%$ \% está informado someramente y un $18.75 \%$ tiene conocimiento del diagnóstico.

Conclusión: En este reporte se evidencia el desconocimiento sobre cáncer de próstata que tienen los encuestados sobre su concepto y diagnóstico.

Palabras Claves: NEOPLASIAS DE LA PRÓSTATA, COMUNICACIÓN, MASCULINIDAD, EDUCACIÓN. 


\section{Abstract}

Introduction: Prostate cancer is a neoplasm in which communication and knowledge of the population can help early diagnosis and early treatment. The objective of this study was to investigate what patients and companions know about the processes of educational communication for health in prostate cancer.

Methods: The present descriptive study was carried out in the National Drugs Institute "Dr Juan Tanca Marengo" Solca-Guayaquil. A health survey was used on the knowledge of the nosological entity, the knowledge of the diagnostic methods and the predisposition to attend educational talks. The sample was calculated in 80 surveys to relatives, companions and patients of the Institute.

Results: 80 surveys were registered. Regarding prostate cancer, $52.5 \%$ of the respondents declare to have no knowledge about it, $31.25 \%$ claim to have little knowledge about prostate cancer, and $16.25 \%$ declare to have an appropriate knowledge about prostate cancer. About the diagnosis of Prostate Cancer, $58.75 \%$ of respondents do not know how it is diagnosed, $22.5 \% \%$ is briefly informed and $18.75 \%$ have knowledge of the diagnosis.

Conclusion: This report shows the lack of knowledge about prostate cancer that respondents have about their concept and diagnosis.

Keywords: PROSTATE NEOPLASMS, COMMUNICATION, MASCULINITY, EDUCATION.

DOI: $10.33821 / 240$

\section{Introducción}

La comunicación desde la prehistoria ha jugado un rol importante para el desarrollo de una sociedad, por esta razón es necesario hacer transformaciones en la forma de comunicar. La comunicación se puede explicar mejor si partimos de la consideración del proceso biosocial del ser humano [1]. La relevancia de este tema se proyecta en la limitada educación, debido a la creación de estereotipos sobre las diferentes formas de prevenir el cáncer prostático. En la actualidad esta enfermedad representa un gran reto social, toda vez que su incidencia aumenta con el desarrollo económico e industrial de los países, así como con el perfeccionamiento de los sistemas de salud [2].

Esto genera que la enfermedad se ubique como una de las primeras causas de mortalidad en hombres. Según registra el Instituto Nacional de Estadística y Censos (INEC) [3], los fallecimientos por año son de 153 personas en Guayaquil; donde el $64 \%$ son hombres mayores de 75 años. La educación y prevención del cáncer de próstata a través de los medios televisivos ayudaría a la detección temprana para evitar fallecimientos por esta causa.

La acción humana no es entelequia que se produzca en el vacío, y los jóvenes que ponen en riesgo su salud en el seno de sus escenarios vitales se basan en una concepción que ampara y legitima esos comportamientos [4]. En este contexto, esta investigación permite evidenciar la importancia sobre el estudio de los procesos comunicacionales educativos en el siglo XXI sobre el cáncer de próstata. A nivel mundial los estudios sobre esta patología confirman que existe una alta probabilidad de que esta enfermedad afecte a los hombres 
menores de 40 años, abriendo cada vez más la brecha sobre la edad y creando un margen mayor de padecimiento después de los 65 años. Esta afección representa la primera causa de mortalidad en los países de la región noroeste de Europa, las islas del Caribe, Australia y Norteamérica. En Asia, África, Centroamérica y en Sudamérica la posibilidad de tener cáncer de próstata es alta, de hecho es la segunda causa de mortalidad en la población masculina. Los aspectos de la prevención en salud que de alguna forma infrinjan la construcción de la masculinidad terminan siendo negados por los hombres sin importar las consecuencias que resulten [5].

En Latinoamérica la detección temprana del cáncer de próstata es muy baja, debido a que los afectados recurren a la revisión cuando la enfermedad está en su último ciclo, es decir, en una etapa avanzada. Dejando en evidencia la necesidad de desarrollar e implementar planes y programas nacionales para el control y la difusión sobre esta enfermedad y sus diferentes pruebas para la prevención, detección temprana, diagnósticos y tratamiento, para concienciar sobre la necesidad de que los hombres se realicen a tiempo un examen antes que las consecuencias sean irreversibles. En Guayaquil, solamente en el 2015 se detectaron 3.801 nuevos casos de pacientes con cáncer. De los cuales 600 fueron de mama, seguidos de 433 de cuello uterino y, por último, 283 con cáncer de próstata.

El objetivo de este estudio fue investigar que conocen los pacientes y acompañantes sobre los procesos de comunicación educativa para la salud en cáncer de próstata.

\section{Materiales y Métodos}

El presente estudio descriptivo fue realizado en diciembre de 2017 en el que se midieron a través de un cuestionario, la información, comunicación, tratamiento, características y la prevención que en materia de cáncer de próstata tenían un grupo de familiares y pacientes de Solca. El cálculo de la muestra fue de 80 casos de encuesta. Esta muestra se llevó a cabo en familiares de los pacientes que fueron entrevistados en los exteriores del Hospital de Solca de Guayaquil. Los criterios de inclusión fueron familiares de pacientes hospitalizados en el Instituto Oncológico Nacional "Dr Juan Tanca Marengo"- Solca Guayaquil y visitantes, escogidos de forma aleatoria, que necesitaron diagnóstico, tratamiento de curación y paliación debido al padecimiento de la enfermedad. Se excluyeron a personas menores de 40 años y mayores de 65 años de edad, pacientes que declararon haber recibido atención en otro centro de salud, mujeres y pacientes en la etapa de curación y paliación.

\section{Resultados}

En el estudio se incluyeron 80 encuestados (Tabla 1). Sobre el cáncer de próstata el $52.5 \%$ de los encuestados declara no tener conocimiento alguno sobre el mismo, un $31.25 \%$ asegura tener poco conocimiento sobre el cáncer de próstata, solo un $16.25 \%$ declara tener un conocimiento apropiado sobre el cáncer de próstata (Figura 1). 
Tabla 1. Participantes en la encuesta

\begin{tabular}{|c|c|c|}
\hline Ítem & Estrato & Muestra \\
\hline $\mathbf{1}$ & Visitantes & 50 \\
\hline $\mathbf{2}$ & Pacientes & 30 \\
\hline & Total & 80 \\
\hline
\end{tabular}

Figura 1. Conocimientos sobre el Cáncer de Próstata

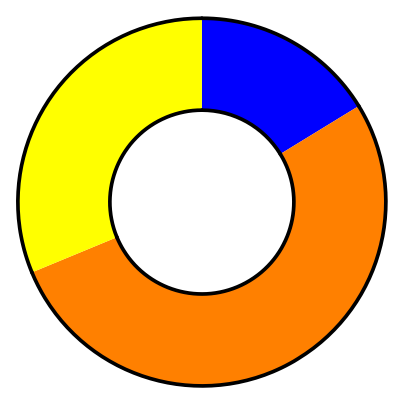

$\square \mathrm{n}=42(52.5 \%)$ Sin conocimientos

$\square \mathrm{n}=25(31.25 \%)$ Pocos conocimientos

$\mathrm{n}=13(16.25 \%)$ Conocimientos apropiados

Total $=80$

Sobre el diagnóstico de Cáncer de Próstata el 58.75 \% de los encuestados no sabe o no quiere decir cómo se diagnostica, un $22.5 \%$ de los encuestados menciona que se ha informado en algún momento su diagnóstico, y un $18.75 \%$ de los encuestados puede entablar un diálogo con referencia al tema (Figura 2).

Figura 2. Conocimientos sobre el Diagnóstico de Cáncerde Próstata

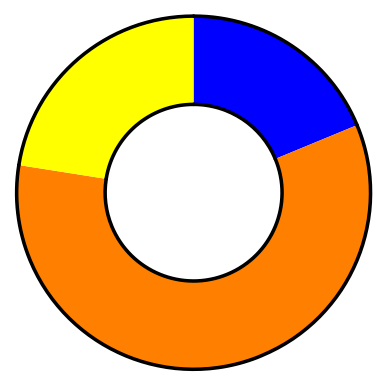

$\square \mathrm{n}=47(58.75 \%)$ No conoce

$\square \mathrm{n}=18(22.5 \%)$ Informado levemente

$\square \mathrm{n}=15(18.75 \%)$ Conocimientos apropiados

Tota $\mathrm{I}=\mathbf{8 0}$

De los encuestados el $52.5 \%$ declaran su intención de asistir a una charla informativa por tener conocimiento de las consecuencias del cáncer de próstata, el $22.5 \%$ de los encuestados declara su intención de asistir a una charla informativa por curiosidad y el 25 \% declara su preferencia de no acudir a ninguna charla informativa (Figura 3). 
Figura 3. Intención de información sobre Cáncer de Próstata
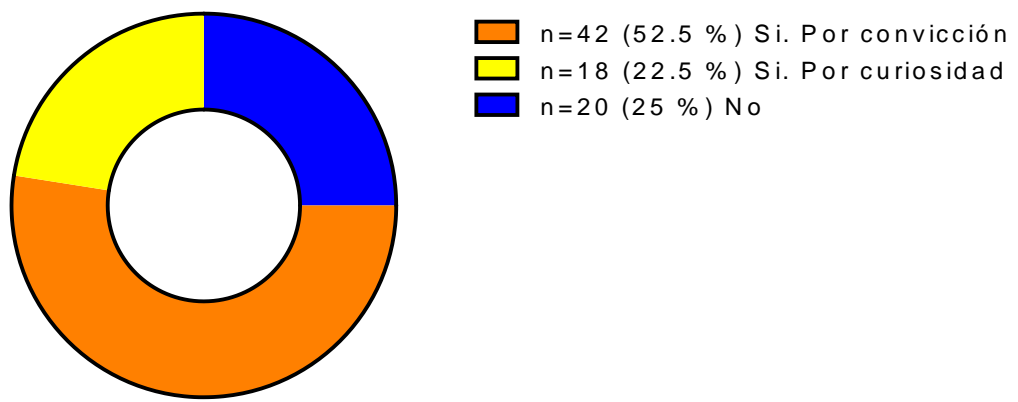

Tota $\mathrm{l}=80$

\section{Discusión}

Durante la investigación se pudo evidenciar el desconocimiento y hermetismo que tienen los hombres al hablar sobre el cáncer de próstata. La información que la institución antes mencionada otorga con base en lo expuesto se enfatiza en él pero solo llega a quienes visitan el lugar directa o indirectamente. Aunque el cáncer de próstata se ha socializa bajo diferentes estrategias aún se refleja que $>50 \%$ no sabe nada sobre ella.

En Ecuador la difusión sobre el cáncer de próstata es menor con relación al cáncer de mama $\mathrm{u}$ otro problema de salud. Esto pese a que el Ministerio de Salud Pública y otras instituciones especializadas como Solca desarrollan y promueven estrategias, planes y programas de información, educación y comunicación social en salud [6]. La Carta de Ottawa para la promoción de la salud indica que por tanto la promoción de la salud no es simplemente responsabilidad del sector sanitario, sino que va más allá de los estilos de vida saludables para llegar al bienestar [7].

Los aspectos de la prevención en salud que de alguna forma terminan siendo negados por los hombres sin importar las consecuencias que resulten [8]. El Instituto Nacional de Estadísticas y Censos (INEC) muestra en sus registros 1.585 actas de defunción con lo que respecta al cáncer, de las que 833 son por Neoplasia de próstata en el 2014. En Ecuador se producen aproximadamente 750 muertes anuales y es la segunda causa de muerte en los varones, y al igual que en muchos países, este tema se maneja de manera superficial, se realizan difusión de campañas sobre la prevención o la importancia de realizarse la prueba a tiempo de forma esporádica. La salud forma parte de la propia vida, parece consustancial con nosotros, se posee, se sirve con ella y nos la cuestionamos, no reflexionamos sobre su significado [9].

Entre las conclusiones que se propone está la de crear producciones audiovisuales sobre el cáncer de próstata, que contenga mensajes positivos y persuasivos. Realizar investigaciones que involucren la parte comunicacional de la familia con respecto a temas de salud como el cáncer. Trabajar mediante convenios en las instituciones que se dedican 
a las investigaciones de salud. Realizar charlas y socializar la información del cáncer de próstata en las unidades educativas. La educación y prevención del cáncer de próstata a través de los medios masivos como la televisión ayudaría a la detección temprana para evitar fallecimiento por esta causa. Se debe "informar, influir y motivar audiencias a nivel individual, institucional y público acerca de asuntos de salud importantes. Su alcance incluye prevención de enfermedad, política de cuidado de salud, así como el aumento de la calidad de vida y salud de los individuos dentro de la comunidad" [9].

Como el objetivo de este estudio fue investigar sobre los procesos de comunicación educativa para la salud sobre el cáncer de próstata en la ciudad de Guayaquil, se pudo demostrar que no es lo mismo informar que crear una cultura de prevención. En cuanto a los contenidos teóricos que se relacionan con los procesos comunicacionales educativos para la salud, la colectividad en general es vocera de los cambios positivos o negativos que se generan a raíz de una campaña, talleres o materiales complementarios. Y al analizar la situación actual de los procesos de difusión de contenidos comunicacionales sobre el cáncer de próstata en hombres de 40-45 años, los ejes en la mayoría han equivocado el rumbo porque de forma general se refieren al papel que el hombre ocupa en la sociedad y la influencia que tiene lo que se diga de él, ya que su rol está jerarquizado como superior, ejemplar y fuerte, es decir a él no se le permite llorar, enfermarse o quejarse. Nuevos estudios serán requeridos en esta área tomando en cuenta conocimientos, actitudes y prácticas pre y post capacitaciones sobre cáncer de próstata a la comunidad.

\section{Conclusiones}

En este reporte se evidencia el desconocimiento sobre cáncer de próstata que tienen los encuestados sobre su concepto y diagnóstico.

\section{Agradecimientos}

Reconocemos a las personas que participaron indirectamente en el estudio tales como el personal técnico, familiares y visitantes del Instituto Oncológico Nacional "Dr Juan Tanca Marengo"- Solca Guayaquil.

\section{Información adicional}

Nota del Editor

La Revista Oncología Ecu permanece neutral con respecto a los reclamos jurisdiccionales en mapas publicados y afiliaciones institucionales.

\section{Abreviaturas}

INEC: Instituto Nacional de Estadísticas y Censos. 
Ninguno declarado por los autores.

Fondos

Los fondos de la investigación fueron propios de los autores del presente artículo.

\section{Disponibilidad de datos y materiales}

Existe la disponibilidad de datos bajo solicitud al autor de correspondencia. No se reportan otros materiales.

\section{Contribuciones de los autores}

DPBV, PVL realizaron la idea de investigación, revisión bibliográfica. JAVF y JCJ, recolección de datos, escritura del artículo. PVL realizó el análisis crítico del artículo. Todos los autores leyeron y aprobaron la version final del artículo.

Aprobación de ética y consentimiento para participar

No aplica a una encuesta.

\section{Consentimiento para publicación}

No aplica.

\section{Información de los autores}

Diana del Pilar Baquerizo Villacís, Lcda. en Ciencias de la Comunicación Social por la Facultad de Comunicación Social (Facso), Universidad de Guayaquil. https://orcid.org/0000-0001-7022-6986

Jhonny Amed Vargas Flores, Profesor de la Facultad de Comunicación Social (Facso) de la Universidad de Guayaquil.

John Chusán Jiménez, Médico. Responsable del Centro de Referencia Nacional de Micología en el Instituto Nacional de Investigación en Salud Pública (INSPI). 
Patricio Vega Luzuriaga. Dirección Técnica de Transferencia del Conocimiento del Instituto Nacional de Investigación en Salud Pública (INSPI).

Revisiones por pares

Acceda a la revisión de pares académicos en el siguiente enlace: https://publons.com/review/3791785/

\section{Referencias}

1. Guardia de Viggiano N. Lenguaje y comunicación. $1^{\text {a }}$. ed. - San José, C.R.: Coordinación Educativa y Cultural Centroamericana, CECC/SICA, 2009;25:1-140. (Colección Pedagógica Formación Inicial de Docentes Centroamericanos de Educación Básica; n. 25). SU: goo.gl/KzfRfj

2. Fernández M, Corona L, Hernández J, Espinosa A, Pereira E, Fuigueiras B. Mortalidad por neoplasias malignas en la población adulta de la provincia de cienfuegos durante el decenio 1988-1997. Rev Cubana Med 2003;42(2):23-29. SU: goo.gl/cbe7Jk

3. INEC: Instituto Nacional de Estadísticas y Censo (Internet). Ecuador: INEC; c2018 (citado en noviembre de 2017) Disponible en: ecuadorencifras.gob.ec.

4. Aguilera Moyano, M, Pindado Pinado, J. Nuevos enfoques en comunicación y salud: perspectivas de investigación. Comunicar 2006;(26):13-20. SU: goo.gl/KZrYHE

5. Abou Orm Saab, K, Camacaro Cuevas, M. Determinantes socioculturales que condicionan la masculinidad y su impacto en la salud sexual y reproductiva de los hombres. Comunidad y Salud 2013;11(1):25-34. SU: goo.gl/MN6rVo

6. Congreso Nacional. LEY ORGANICA DE SALUD. Registro Oficial Suplemento 423 de 22-dic.-2006. Art 3, 6, 12. 2012. SU: goo.gl/Rqqzx3

7. Otawa C. Carta De Ottawa Para la promoción de la Salud. Salud Pública Educ Salud, (19-22). 1986. SU: goo.gl/twicWa

Abreviaturas en la referencias

DOI: Digital Object

Identifier

PMID: PubMed Identifier

SU: Short URL
8. Gavidia V. Talavera M. La construcción del concepto de salud. Dpto. Didáctica CC. Experimentales y Sociales. 2012. 26, 161-175. DOI: 10.7203/DCES.26.1935

9. Martinezbeleño C, Sosa Gomez M. Aportaciones y diferencias entre comunicación en salud, comunicación para el desarrollo y para el cambio social. Revista de comunicación y Salud, 2016;6:6980. SU: goo.gl/1NeSjx 